\title{
Criterion level and task difflculty as determinants of reversal learning
}

THOMAS W. TURNAGE AND JENNY I. STEINMETZ,1 DEPARTMENT OF PSYCHOLOGY, UNIVERSITY OF MARYLAND, College Park, Maryland 20740

Reversal learning was investigated in 42 children with two levels of stimulus discriminability (Easy and Difficult) and three acquisition criteria $(50 \%, 75 \%, 100 \%)$. Ease of reversal learning, as predicted by $(a)$ the ratio of number of rewarded trials to total trials during acquisition $\left(r_{e}\right)$ or $(b)$ the criterion level required during acquisition, was positively correlated with degree of acquisition trainting. Manipulation of stimulus discriminability did not affect reversal.

Using children, Steinmetz \& Turnage (1966) found (a) that ease of reversal learning is directly related to a ratio of number of reinforced trials to total number of trials, which measure they called $r_{e}$, and (b) that introducing a novel $S^{D}$ on reversal did not affect reversal rate. Such results seemed hard to reconcile with various explanations of the Overlearning Reversal Effect (ORE), with respect to their emphases on the critical importance of avoidance tendencies to the $S^{d}$ (D'Amato \& Jagoda, 1961) and discrimination of a problem change (Capaldi \& Stevenson, 1957). However, these results did support the interpretation of Birch, Ison, \& Sperling (1960) that overtraining facilitates reversal learning by leading to faster extinction of the previously correct response. When this is considered along with (a) Jenkin's (1961) finding that something akin to a partial reinforcement schedule is generated in successive discrimination studies and (b) the knowledge that the reinforcement schedule during acquisition is an important determinant of rate of extinction (cf., Ferster \& Skinner, 1957), an important implication emerges. That is, reversal rate-as suggested by its direct relation to $r_{0}$-should be influenced by the difficulty of discrimination learning. More particularly, the given "schedule" of reinforcement generated ("CRF" vs "Intermittent") should be directly related to the difficulty of the discrimination, since the faster the $S$ learns a discrimination the faster he approximates a CRF schedule for himself. This in turn should facilitate reversal learning. Unfortunately, there is very little direct experimental evidence on this point with respect to reversal learning, although Hoffman, Perkins, \& Calvin (1956) found that rats mastered both a discrimination and subsequent reversal faster if the two grays used differed more in brightness than a comparison pair. Consequently, the present experiment was designed to investigate directly the discriminability of experimental stimuli as a parameter of $r_{e}$. An additional point considered was the precision of prediction when $r_{e}$ was computed using only the block of criterion trials as compared to using all of the acquisition trials.

Method

Subjects. The Ss were 42 boys, ranging in age from six to nine (mean, 7.3 years), attending a weekly clinic conducted by the Physical Education Department at the University of Maryland. ${ }^{2}$ They had been referred by the particular public schools which they attended as needing special education in motor and/or social skills, but all were of average or above average intelligence (estimated range, 90-145; mean, 114.9).

Apparatus and Stimuli. An automated apparatus, especially constructed for this experiment, required that the $S$ face a small screen which was flanked by a lever on each side. The $S^{D}$ and $S^{d}$ were projected simultaneously on the screen from the rear by a Carousel slide projector which was activated by depressing either, but not both, of these levers.

The stimuli used were selected from a set described by Gibson (1963) as appropriate for the ages represented in this study. One symbol from a set characterized as "perspective transformation" was reported by her as difficult to distinguish from a test symbol, while one symbol classified as "break and close" was reported to be easy to discriminate from this test symbol. Therefore, the first stimulus combination was designated the difficult task (D) and the second combination the easy discrimination task (E) for the present study.

Procedure. The design was a 2 by 3 factorial with two levels of discrimination (E and $D)$ and three acquisition criteria $(50 \%, 75 \%$, and $100 \%$ ). Seven Ss were arbitrarily assigned to one of the six experimental conditions, with the restriction that all six experimental conditions were to be used within each successive block of six Ss. All Ss were tested individually. The order for position of stimuli (right vs left) was determined by a Fellows (1967) series. In each experimental condition four $S$ s received one stimulus as the $S^{D}$, and three Ss received the other.

All Ss were initially trained without the $S^{D}$ and $S^{d}$ to a left or right position habit (five consecutive responses to a given side). Immediate reinforcement for a correct response was a buzzer. When the buzzer sounded, $S$ took a checker which could be traded at the end of the experiment for trinkets of the variety seen in gum ball machines.

After the position habit was established, $\mathrm{S}$ was asked to look at the screen and push the correct lever below the "pictures" (experimental stimuli). $\mathrm{E}$ informed him that if he guessed right the buzzer would sound, and he could take another checker. After every response, there was a 5-sec period during which the stimuli were still present, but neither lever was operative. At the end of 5 -sec a new slide appeared which might show the stimuli in the same position or in the reverse one.

Training was terminated for the 50\% group after 12 trials while the Ss were still responding at chance level, since in an earlier study (Steinmetz \& Turnage, 1966) this number had been determined appropriate to ensure that acquisition training would end while Ss were still responding equally to the two stimuli. Acquisition training ended for Ss in the $75 \%$ group when any 9 of 12 consecutive responses were correct. Criterion for the $100 \%$ group was 10 consecutive correct responses.

Approximately $30 \mathrm{~min}$ after termination of acquisition training, the reversal test began. During the time when $S$ was not in the experimental room, he was engaging in his regular gym activities. The procedure for reversal learning was the same as that for acquisition trials. The $S$ was told that he was again to press the correct lever under the picture on the screen. Criterion for successful reversal for all Ss was 8 correct responses out of any 10 consecutive trials. At the end of each session, acquisition and reversal, Ss were allowed to choose four trinkets, regardless of the number of checkers earned.

Results and Discussion

Acquisition. Table 1 shows the mean number of trials to criterion blocks for the six experimental groups for both acquisition and reversal. The monotonic relationship between criterion level and acquisition trials for the $D$ groups as opposed to the nonmonotonic one for the $E$ groups indicated a significant interaction. Because the initial training procedure produced a variance of zero for the two $50 \%$ groups, as well as identical means, the data in Table 1 could not be statistically evaluated by an analysis of variance involving all six groups. However, an analysis of the acquisition trials for the four groups with variance indicated a significant Task Difficulty (TD) by Criterion Level (CL) interaction, $F(1,24)=6.99, p,<.05$, but showed that neither of the main effects was significant, $\operatorname{Fs}(1,24)<3.75$. The Scheffé test for post hoc comparisons (Hays, 1963) indicated that the $75 \% \mathrm{D}$ and $100 \% \mathrm{D}$ groups differed significantly at the .05 level, while the $75 \%$ and $100 \% \mathrm{E}$ groups did not. These comparisons along with the configuration of means shown in Table 1 indicated that the difficult discrimination had a linear trend across criterion levels, while the easy discrimination had a nonlinear trend. This outcome will be discussed subsequently in a more general context, but at this point we turn to an analysis of the main focus of the experiment-the reversal data. 
Table 1

Trials to Criterion Block for the Easy and Difficult Discrimination Conditions During Acquisition and Reversal

\begin{tabular}{llllllll}
\hline & \multicolumn{2}{c}{\begin{tabular}{c}
\multicolumn{2}{c}{ Acquisition } \\
\end{tabular}} & \multicolumn{2}{c}{ Criterion level } & & \multicolumn{3}{c}{$\begin{array}{c}\text { Reversal } \\
\text { Criterion level }\end{array}$} \\
\cline { 2 - 6 } & $50 \%$ & $75 \%$ & $100 \%$ & & $50 \%$ & $75 \%$ & $100 \%$ \\
Easy & 0 & 15.29 & 12.14 & & 16.43 & 14.00 & 3.43 \\
Difficult & 0 & 10.29 & 30.57 & & 25.43 & 18.00 & 6.00 \\
\hline
\end{tabular}

Reversal. Table 1 also shows reversal trials as a function of TD and CL. An analysis indicated that CL was significant as predicted, $F(2,36)=5.02, p<.05$, but contrary to expectation, neither the TD effect nor its interaction with $C L$ reached the .05 level of significance, $F_{S}<1.45$. A strength of association measure, $w^{2}$ (Hays, 1963), indicated that $16 \%$ of the variance among Ss was attributable to $\mathrm{CL}$. Thus, $r_{\mathrm{c}}$ based solely on the block of criterion trials was a reliable predictor of speed of reversal learning which confirms a previous finding under comparable conditions (Steinmetz \& Turnitge, 1966).

An $r_{e}$ was computed for each $S$ over all his acquisition trials, viz, we divided total trials into number of reinforced trials. The means of $r_{\mathfrak{c}}$ for the $50 \%, 75 \%$, and $100 \% \mathrm{E}$ groups were, respectively, $.490, .595$, and .745 ; while for the $\mathrm{D}$ groups they were $.512, .592$, and .622 . At this point the predictive value of $r_{c}$ was considered in two additional ways. First, the measure was related to trials to reversal criterion and found to be significant as hypothesized, $r(40)=-.40, p<.01$. This outcome is consistent with the finding of a significant $C L$ effect as discussed above and with the general analysis of the predictive power of $r_{e}$. Second, we computed the correlation between $r_{e}$ and reversal trials for the $E$ group, $r=-.67$, and for the $D$ group. $r=-.53$, in order to evaluate further the effect of discriminability on the predictive accuracy of $r_{c}$. Both correlations differed significantly from 0.00 at the .01 level, but neither differed significantly from the total group correlations nor from each other, $z s<1.15$. Thus, $r_{e}$ determined either by criterion level or computed over all trials accurately predicted reversal rate. but did not interact reliably with the difficulty of the discrimination.

The results of this study support the earlier Steinmetz \& Turnage (1966) finding that $r_{\mathrm{c}}$ is an accurate predictor of reversal rate, and our general findings are again most consistent with the Birch et al (1960) explanation of the ORE.

Stimulus Similarity and the ORE. There seems little doubt that the two stimulus sets used differed in ease of discriminability during acquisition, presumably due to differences in physical similarity. On the other hand, there was no reliable statistical evidence that TD affected reversal rate, which is puzzling in terms of many interpretations of discrimination learning utilizing concepts such as generalization (cf., Spence, 1937). However, the trends for the reversal data (see Table 1) are as might be expected by such theoretical accounts, viz, reversal was always absolutely faster, on the average, for the $E$ groups. Be that as it may, rate of reversal learning was reliably related to $r_{e}$ as predicted.

Reinforcement Differential and ORE. Two factors seem of prime importance in the ORE. One which is amply discussed in the literature (Sperling, 1965; Mackintosh, 1965) is a species predisposition to respond to certain types of perceptual cues. The other which does not seem as well appreciated, and is suggested by studies such as ours, is the necessity of a high differential pattern of reinforcement among experimental groups. Let us examine some cases in point.

First, if the task is too "casy" and is learned too quickly, the reinforcement differential among groups will not be meaningful in terms of the behavioral consequences, and of course differences in $r_{c}$ will be numerically small. An example of this was furnished by a recent pilot study in which length of line was the relevant dimension. The discrimination was learned to the $100 \%$ criterion by 6 out of 8 Ss in 12 trials. and all Ss met the reversal criterion of $80 \%$ within 12 trials. There was no ORE, of course. Second, failures of ORE may also be attributed to procedures which attenuate the reinforcement differential in other ways. D'Amato \& Jagoda (1962) noted that one difference between experiments in which the ORE was found and those where it was not was in the use of forced trials to the $S^{d}$ in the latter case. These forced trials were essential to their position concerning the aversive characteristic of $S^{d}$, but as Birnbaum (1961) noted such studies maintain an "intermittent" schedule of reinforcement which may retard extinction of responding to the old $S^{D}$ during reversal. That is, some of the time preceding reversal (for both the criterion and overtrained groups), $S$ was forced to approach $S^{d}$ and to receive no reinforcement. Thus, there was little difference in pattern of reinforcement, and $r_{e}$ probably was roughly equivalent for both groups. It is also pertinent that Lewis (1960) reported that partial reinforcement tends to retard reversal rate. Third, many ORE studies have trained all Ss to rather stringent criteria, and then proceeded with overtraining. Thus, all such groups are on something approximating a CRF schedule before criterion is reached. This would tend to equate groups in terms of reinforcement differential (and consequently $\mathrm{r}_{\mathrm{e}}$ ); and, thereby, reduce the probability of demonstrating the ORE according to this analysis. It should be noted that these remarks may also apply to the present study in that a large reinforcement differential did not obtain between the $E$ and $D$ groups, the average $r_{e}$ computed across all trials being .610 and .575 for the $E$ and $D$ groups, respectively. For this reason the relation between TD and the ORE may have been masked. However, it still seems reasonable to consider the ORE basically from a pattern of reinforcement point of view, and, obviously, this is the basis of the $r_{e}$ index.

\section{REFERENCES}

BIRCH, D., ISON, J. R., \& SPERLING, S. E. Reversal learning under single stimulus presentation. J. exp. Psychol., 1960, 60, 36-40.

BIRNBAUM, I. M. The effects of overlearning on discrimination reversal. Unpublished Manuscript, 1961.

CAPALDI, E. J., \& STEVENSON, H. W. Response reversal following different amounts of training. J. comp. physioL Psychol, 1957, 50, 195-198.

D'AMATO, M. R., \& JAGODA, H. Analysis of the role of overlearning in discrimination-reversal. J. exp. Psychol., 1961, 61, 45-50.

D'AMATO, M. R., \& JAGODA, H. Overlearning and position reversal. J. exp. Psychol, 1962, 64, 117-122.

FELLOWS, B. J. Chance stimulus sequences for discrimination tasks. Psychol Bull., 1967, 67, 87-92.

FERSTER, C. B., \& SKINNER, B. F. Schedules of reinforcement. New York: Appleton-Century-Crofts, Inc., 1957.

GIBSON, E. J. Development of perception: discussion of depth compared with use of graphic symbols. Monogr. Res. Child Develpm., 1963, 28, 5-23.

HAYS, W. L. Statistics for psychologists. New York: Holt, Rinehart and Winston, 1963.

HOFFMAN, F. K., PERKINS, M. J., \& CALVIN, A. The effect of problem difficulty on discrimination reversal J. comp. physiol. Psychol., 1956, 49, 547-548.

JENKINS, H. M. The effect of discrimination training on extinction. J. exp. Psychol., 1961, 61, 111-121.

LEWIS, D. J. Partial reinforcement: a selective review of the titerature since 1950. Psychol Bull., 1960, 57, 1-28.

MacKINTOSH, N. J. Selective attention in animal discrimination learning. Psychol Bull., 1965, 64, 124-150.

SPENCE, $K$. W. The differential response in animals to stimuli varying within a single dimension. Psychol. Rev., 1937, 44, 430-444.

SPERLING, S. E. Reversal learning and resistance to extinction: a review of the rat literature. Psychol Bull., 1965, 63, 281-297.

STEINMETZ, J. l., \& TURNAGE, T. W. An empirical index for ease of reversal learning. Psychon. Sci., 1966, 6, 467-468.

\section{NOTES}

1. This paper is based on a thesis submitted by JIS in partial fulfillment of the M. A. degree in the Department of Psychology at the University of Maryland. The research was partially supported by a Public Health Fellowship, 1-F1-MH37, from the National Institute of Mental Health awarded to her.

2. The cooperation of Dr. and Mrs. Warren Johnson and the staff of the Physical Development (Clinic of the University of Maryland is gratefully acknowledged. 